\title{
Preoperative biliary drainage versus direct surgery for perihilar cholangiocarcinoma: A retrospective study at a single center
}

\author{
Yulong Cai ${ }^{1}$, Qi Tang ${ }^{2}$, Xianze Xiong ${ }^{1}$, Fuyu Li ${ }^{1}$, Hui Ye ${ }^{1}$, Peipei Song ${ }^{3}$, Nansheng Cheng ${ }^{1, *}$ \\ ${ }^{1}$ Department of Bile Duct Surgery, West China Hospital, Sichuan University, Chengdu, Sichuan, China; \\ ${ }^{2}$ Department of Social Medicine and Medical Service Management, School of Public Health, Shandong University, Ji'nan, \\ Shandong, China; \\ ${ }^{3}$ Graduate School of Frontier Sciences, The University of Tokyo, Kashiwa-shi, Chiba, Japan.
}

\begin{abstract}
Summary Perihilar cholangiocarcinoma (pCC, also known as a Klatskin tumor) is the most common type of cholangiocarcinoma (CC). Preoperative biliary drainage (PBD) is indicated for pCC patients with acute cholangitis or patients who need portal vein embolization (PVE). However, the routine performance of $\mathrm{PBD}$ in other patients with $\mathrm{pCC}$ is still controversial. The current study retrospectively examined patients with $\mathrm{pCC}$ who did not undergo PVE and who did not have cholangitis who were seen at this Hospital to assess the advantages and disadvantages of PBD. This study also sought to find an optimal value of total bilirubin (TB) to indicate performing PBD. Between 2009 and 2014, after excluding patients with acute cholangitis and PVE, patients who had undergone hepatectomy for pCC were enrolled in this study. First, the surgical outcomes and postoperative outcomes were compared between PBD group and direct surgery group. Second, ROC curve analysis of a subgroup of patients was performed to find the best cut off value of TB for indicating the PBD. Third, the costs for patients, including the total charges and the charges per day were compared between the two groups. Subjects were 218 patients in total. Fifty-five patients underwent PBD. This group had a longer operative time [390 (210-700) vs. 360 (105-730) $\mathrm{min}, p=0.013]$, and a longer hospital stay [20 (9-48) vs. 17 (6-93) days, $p=0.007]$, but underwent vascular resection and reconstruction less often [8 (14.5\%) vs. $50(30.7 \%), p=0.019$ ]. Mortality and morbidity were comparable between the two groups. ROC curve analysis of a subgroup of patients indicated that the cut-off value for total bilirubin was $218.75 \mu \mathrm{mol} / \mathrm{L}(12.4 \mathrm{mg} / \mathrm{dL})$. The total hospital charges and the charges per day did not differ significantly for the two groups. Disadvantages of PBD were a longer operating time and a longer duration of hospitalization, but the short-term surgical outcomes and hospital charges of PBD group were comparable to the direct surgery group. PBD should be considered for patients when the diagnosis is still suspicious of pCC. Based on the current data, the optimal cut-off value for preoperative TB was $218.75 \mu \mathrm{mol} / \mathrm{L}(12.4 \mathrm{mg} / \mathrm{dL})$ to indicate PBD for patients with pCC.
\end{abstract}

Keywords: Cholangiocarcinoma, Klatskin tumor, preoperative biliary drainage, bilirubin, hospital cost

\section{Introduction}

Perihilar cholangiocarcinoma (also known as a Klatskin

Released online in J-STAGE as advance publication May 22, 2017.

*Address correspondence to:

Dr. Nansheng Cheng, Department of Bile Duct Surgery, West China Hospital, Sichuan University, Chengdu, Sichuan Province, China.

E-mail: nanshengcheng2012@163.com tumor) is the most common type of cholangiocarcinoma (CC). Perihilar cholangiocarcinoma (pCC) is defined as $\mathrm{CC}$ located in the extrahepatic bile duct and involving the confluence of the left and right main hepatic ducts (1). At present, the only potential curative treatment for pCC is surgical resection. In order to achieve an R0 resection (negative margins), a major hepatectomy, in a form such as a right or left trisegmentectomy, must be performed (2). Moreover, patients with pCC are known to have obstructive jaundice. Given this fact, patients 
have higher postoperative morbidity and mortality rates due to hepatic insufficiency and infections (3).

Preoperative biliary drainage (PBD) and portal vein embolization (PVE) are used for preoperative optimization of the liver. PBD is performed to prevent cholestasis-associated toxicities and PVE is performed to improve regeneration of a small future remnant liver (FLR) (4). According to current evidence and guidelines regarding $\mathrm{pCC}$, biliary drainage is definitely indicated for a patient with pCC and acute cholangitis, but the routine performance of biliary drainage is controversial (5). In addition, PBD is recommended for patients who are eligible for PVE (6).

A meta-analysis has indicated that PBD provided no advantage when treating a tumor causing obstructive jaundice (7). Therefore, whether PBD should be undergone by patients without acute cholangitis and who do not need PVE is still controversial. Moreover, surgeons usually choose to perform PBD depending on the level of total bilirubin (TB), but few studies have examined an appropriate value for TB to indicate the need for PBD.

The current study retrospectively examined patients with pCC who did not undergo PVE and who did not have cholangitis who were seen at this Hospital to assess the advantages and disadvantages of PBD. This study also sought to determine an optimal value for TB to indicate the need for PBD.

\section{Materials and Methods}

\subsection{Patients}

Potential subjects were patients had undergone hepatectomy for pCC between 2009 and 2014 at a tertiary care facility (Department of Bile Duct/Pancreat/ Liver Surgery, West China Hospital, Sichuan University, China). Patients with acute cholangitis and patients who underwent PVE were excluded, resulting in 218 patients in total. The clinical records of these patients were retrospectively reviewed using the Hospital's database. pCC was confirmed based on pathological findings in all patients. Fifty-five patients underwent PBD and the remaining 163 patients directly underwent surgery. Patients who died or who hadserious complications (Dindo-ClavienIII-IV) causing hospitalization forover 30 days were excluded to identify a subgroup of patients with a good prognosis postoperatively $(n=184)$. A receiver operating characteristic (ROC) curve analysis of this subgroup of patients was performed. In general, patients were routinely followed for 90 days to analyze the 90 -day mortality and morbidity.

\subsection{Study design}

This study was divided into three parts. First, the outcomes of patients who underwent PBD and those who did not were compared to assess the advantages and disadvantages of PBD. Second, ROC curve analysis of a subgroup of patients was performed to find the optimal value for TB to indicate PBD. Third, the cost for patients, including the total charges and the charges per day, were compared for patients who underwent PBD and patients who did not.

\subsection{Definitions}

PBD was defined as insertion of a biliary drain for percutaneous transhepatic drainage (PTBD) or endoscopic biliary drainage (EBD). Patients underwent surgery one week after PBD, and patients who directly underwent surgery usually did so two weeks after they were diagnosed with pCC. The Bismuth-Corlette classification was used to gauge the extent of pCC (8). The American Society of Anesthesiologists (ASA) Physical Status classification system was used to evaluate a patient's physical state before undergoing surgery. Bilirubin levels were measured in units of $\mu \mathrm{mol} /$ $\mathrm{L}(1 \mathrm{mg} / \mathrm{dL}=17.1 \mu \mathrm{mol} / \mathrm{L})$. Postoperative mortality was defined as any death occurring within 90 days of surgery. R0 resection was considered curative treatment when the resection margin was free of the tumor according tomicroscopy. Postoperative morbidity included grade I to IV complications according to the DindoClavienclassification (9). Tumors were identified a massforming, periductal-infiltrating, and intraductal-growing types based on the classification proposed by the Liver Cancer Study Group of Japan (10).

The surgical procedure depended on the location of the tumor. Generally, a right/left hepatectomy with caudate resection, lymph node dissection, common bile duct resection, and Roux-en-Y biliary-enteric anastomosis was performed. Simple extrahepatic bile duct resection was performed only for patients with Bismuth-Corlette type I pCC. The initial rationale for surgery was to achieve an $\mathrm{R} 0$ resection. The total cost for patients included the hospital basic fee and the cost of medications, surgery, and anesthesia. In China, National Health Insurance covers most of the costs, but costs for some surgical instruments are borne by patients themselves.

\subsection{Statistical analysis}

Statistical analyses were performed using SPSS (version 19.0, SPSS Inc. Chicago, IL, USA). Continuous variables with a normal distribution were expressed as the mean \pm standard deviation (SD) and compared using an independent Student's $t$ test. Other continuous variables were expressed as the median (range) and compared using the Mann-Whitney $U$ test. Categorical data were expressed as the number (percentage) and compared using a chi-square or Fisher's exact test as appropriate. ROC curve analysis was used in the subgroup with a 
good prognosis to determine the best cut-off value for TB to indicate the need for PBD. Sensitivity, specificity, positive predictive values (PPV), and negative predictive values (NPV) were calculated for the cut-off point. A $p$ value $<0.05$ was deemed to be significant.

\section{Results}

\subsection{PBD versus direct surgery}

The flowchart in Figure 1 shows how patients were selected. Patients with acute cholangitis and who underwent PVE were excluded, resulting in a total of 218 patients. Fifty-five patients underwent PBD before surgery and 163 patients directly underwent surgery. The demographic and clinical characteristics of the two groups are shown in Table 1. There were no significant differences between PBD group and direct surgery group in terms of age, gender, BMI, smoking, infection with the hepatitis B virus, Bismuth-Corlette classification, or ASA physical status score. In regard to preoperative laboratory data, Aspartate transaminase (AST) levels in the two groups were similar. The PBD group had higher levels of total bilirubin before PBD [281.1 (8.2-565.4) vs. $161.0(8.4-455.6), p=0.000]$ and lower levels of Alanine aminotransferase (ALT) [81 (13-383) vs. 113 (13-765), $p$ $=0.032]$. And the direct surgery group had lower levels of albumin (ALB) [35.7 (28.1-50.1) vs. 37.7 (22.2-71.0), $p=0.029$ ]. Importantly, after patients underwent PBD, the total bilirubin (TB) level one day before surgery did not differ significantly between the two groups [140.9 (7.2-497.4) vs. 161.0 (8.4-455.6), $p=0.467]$.

The surgical and postoperative outcomes are shown in Table 2. The PBD group associated with longer operative time [390 (210-700) vs. 360 (105-730) $\mathrm{min}, p=$ $0.013]$ and hospital stay [20 (9-48) vs. 17 (6-93) days, $p=$ 0.007]. Although there were no deaths in the PBD group, mortality did not differ significantly between the two groups [0 (0\%) vs. 7 (4.3\%), $p=0.263]$. Morbidity was higher in the PBD group but did not differ significantly from that in the other group. Interestingly, fewer patients in the PBD group underwent vascular resection and reconstruction in comparison to the direct surgery group [8 patients $(14.5 \%)$ vs. $50(30.7 \%), p=0.019]$. Total

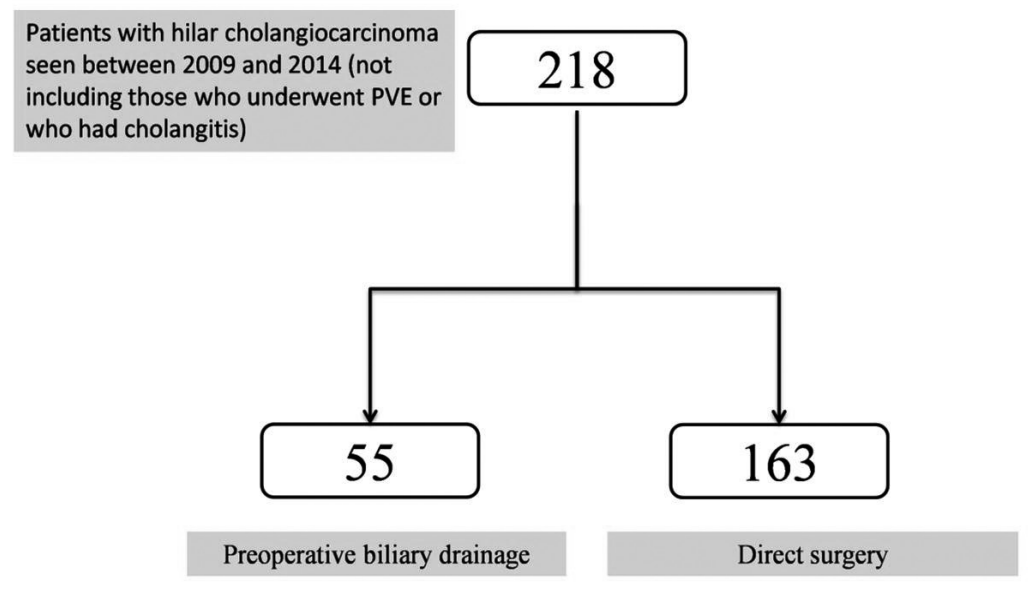

Figure 1. Flowchart of the included participants. PVE, portal vein embolization.

Table 1. Demographic and clinical characteristics

\begin{tabular}{lccc}
\hline Variables & PBD $(n=55)$ & Direct surgery $(n=163)$ & $p$ value \\
\hline Age (years) & $59 \pm 11$ & $60 \pm 9$ & 0.349 \\
Gender (male/female) & $30 / 25$ & $74 / 89$ & 0.240 \\
BMI & $21.8 \pm 5.9$ & $22.5 \pm 6.8$ & 0.519 \\
Smoking & $20(36.4 \%)$ & $65(39.9 \%)$ & 0.644 \\
HBV infection & $5(9.1 \%)$ & $9(5.5 \%)$ & 0.538 \\
Bismuth-Corlette classification & & & 0.469 \\
I/ II/ III//IIIB/ IV & $9 / 13 / 10 / 8 / 5$ & $24 / 28 / 24 / 42 / 45$ & 0.189 \\
ASA physical status score & $2 / 26 / 26 / 0 / 1$ & $1 / 83 / 78 / 0 / 1$ & 0.029 \\
I/II/III/IV/V & $35.7(28.1-50.1)$ & $37.7(22.2-71.0)$ & 0.032 \\
ALB (g/L) & $81(13-383)$ & $113(13-765)$ & 0.114 \\
ALT (U/L) & $74(24-255)$ & $89(14-836)$ & 0.000 \\
AST (U/L) & $281.1(8.2-565.4)$ & $161.0(8.4-455.6)$ & 0.467 \\
T-Bil before PBD $(\mu \mathrm{mol} / \mathrm{L})$ & $140.9(7.2-497.4)$ & $161.0(8.4-455.6)$ & \\
T-Bil after PBD $(\mu \mathrm{mol} / \mathrm{L})$ & &
\end{tabular}

Age and BMI are expressed as the mean \pm standard deviation; other variables are expressed as the number (\%) or median (range). PBD, preoperative biliary drainage; ALB, albumin; ALT, alanine aminotransferase; AST, aspartate transaminase; T-Bil, total bilirubin. 
Table 2. Surgical and postoperative outcomes

\begin{tabular}{|c|c|c|c|}
\hline Variables & $\operatorname{PBD}(n=55)$ & Direct surgery $(n=163)$ & $p$ value \\
\hline \multicolumn{4}{|l|}{ Surgical procedure, $n(\%)$} \\
\hline Right or extended right hepatectomy & $29(52.7 \%)$ & $71(43.6 \%)$ & 0.238 \\
\hline Left or extended left hepatectomy & $17(30.9 \%)$ & $68(41.7 \%)$ & 0.155 \\
\hline Simple bile duct resection & $9(16.4 \%)$ & $24(14.7 \%)$ & 0.769 \\
\hline Operative time, min & $390(210-700)$ & $360(105-730)$ & 0.013 \\
\hline Operative bloody loss, $\mathrm{ml}$ & $500(100-2000)$ & $500(50-3000)$ & 0.324 \\
\hline Transfusion & $26(47.3 \%)$ & $57(35.0 \%)$ & 0.104 \\
\hline Vascular reconstruction & $8(14.5 \%)$ & $50(30.7 \%)$ & 0.019 \\
\hline \multicolumn{4}{|l|}{ Morbidity Dindo-Clavien } \\
\hline Total & $26(47.2 \%)$ & $56(34.4 \%)$ & 0.087 \\
\hline Grade I and II, n (\%) & $13(23.6 \%)$ & $35(21.5 \%)$ & 0.738 \\
\hline Grade III and IV, n (\%) & $13(23.6 \%)$ & $21(12.9 \%)$ & 0.057 \\
\hline Mortality & $0(0.0 \%)$ & $7(4.3 \%)$ & 0.263 \\
\hline Second laparotomy & $1(1.8 \%)$ & $10(6.1 \%)$ & 0.364 \\
\hline Tumor size, cm & $2.9(1.0-6.0)$ & $3.0(0.8-8.0)$ & 0.987 \\
\hline \multicolumn{4}{|l|}{ Surgical margin } \\
\hline R0/R1/R2 & $42(76.4 \%)$ & $125(76.6 \%)$ & 0.961 \\
\hline \multicolumn{4}{|l|}{ Tumor type } \\
\hline $\begin{array}{l}\text { Periductal-infiltrating/ mass-forming/intraductal- } \\
\text { growing }\end{array}$ & $39 / 11 / 5$ & $101 / 44 / 18$ & 0.482 \\
\hline T-Bil $(\mu \mathrm{mol} / \mathrm{L})$ & $180.6(17.0-540.8)$ & $120.2(6.2-461.0)$ & 0.000 \\
\hline POD 3 & $106.5(7.0-601.1)$ & $75.4(8.6-299.6)$ & 0.004 \\
\hline \multicolumn{4}{|l|}{ POD 7} \\
\hline $\operatorname{ALT}(\mathrm{U} / \mathrm{L})$ & $160(17-5524)$ & $187(21-1753)$ & 0.235 \\
\hline POD 3 & $70(12-336)$ & $67(8-1576)$ & 0.843 \\
\hline \multicolumn{4}{|l|}{ POD 7} \\
\hline AST (U/L) & $102(24-24804)$ & $118(23-4528)$ & 0.646 \\
\hline POD 3 & $55(29-394)$ & $47(14-1591)$ & 0.382 \\
\hline \multicolumn{4}{|l|}{ POD 7} \\
\hline $\operatorname{ALB}(g / L)$ & $29.1(21.3-38.3)$ & $29.8(20.0-163.0)$ & 0.355 \\
\hline POD 3 & $31.0(21.2-86.0)$ & $32.8(20.1-67.0)$ & 0.031 \\
\hline POD 7 & $20(9-48)$ & $17(6-93)$ & 0.007 \\
\hline Total hospital stay & & & \\
\hline
\end{tabular}

T-Bil, ALT, AST, and ALB levels for 7 patients who died were excluded. POD, postoperative day.

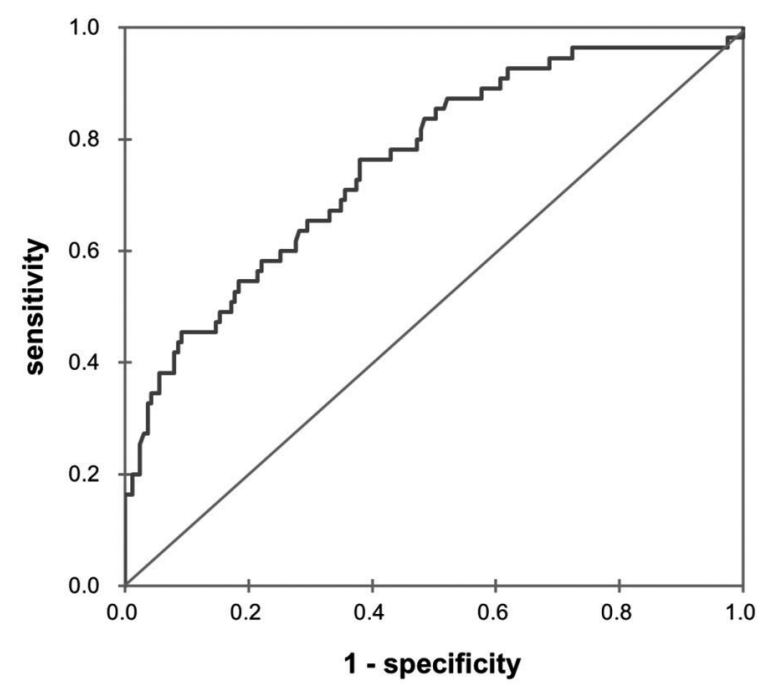

Figure 2. Results of ROC curve analysis of preoperative total bilirubin to predict the need for preoperative biliary drainage in a subgroup of patients. Cut-off value: 218.75 $\mu \mathrm{mol} / \mathrm{L}$; ROC area: 0.780; 95\%CI: 0.699-0.862; Sensitivity (\%): 70.5; Specificity (\%): 72.1; PPV (\%):44.3; NPV (\%): 88.6.

bilirubin was higher on POD 1 and POD 3 in the PBD group, and ALB was lower on POD 7. The surgical procedure, operative blood loss, number of transfusions needed, whether a second laparotomy was performed, tumor size, R0 resection, tumor type, and AST and ALT levels on POD 1 and 7 were similar for the two groups.

The current findings revealed that the PBD group and the direct surgery group had nearly the same postoperative outcomes. Patients in the PBD group had a longer operating time and longer duration of hospitalization but underwent vascular resection and reconstruction less often. There were no deaths in the group that underwent PBD, but mortality did not differ significantly from the other group according to the current findings.

\subsection{ROCcurve analysis}

ROC curve analysis of a subgroup of patients was performed. In order to find the optimal reference value of TB to indicate the need for PBD, patients who died and patients who had major complications that caused hospitalization for over 30 days were excluded. This subgroup of patients had a good prognosis and they can be regarded as right and standard choice of performing PBD on pCC patients. This subgroup consisted of 184 
Table 3. Financial outcomes in the PBD and direct surgery groups

\begin{tabular}{lcc}
\hline Variables & PBD $(n=55)$ & Direct surgery $(n=163)$ \\
\hline Total charge for the treatment & & $p$ value \\
RMB & $93,946 \pm 40,198$ & $84,223 \pm 56,759$ \\
(US\$) & $(14,270 \pm 6,106)$ & $(12793 \pm 8,621)$ \\
Charge per day & & \\
RMB & $4,096 \pm 1,526$ & $4,738 \pm 2,082$ \\
(US\$) & $(622 \pm 231)$ & $(719 \pm 316)$ \\
\hline
\end{tabular}

Cost was calculated based on an exchange rate of 6.6 RMB to 1US dollar and is expressed as the mean \pm standard deviation.RMB is the Ren Min $\mathrm{Bi}$, or the official currency of the People's Republic of China.

patients. Results of ROC curve analysis are shown in Figure 2. The cut-offvalue for total bilirubin was $218.75 \mu \mathrm{mol} / \mathrm{L}$, with a sensitivity of $70.5 \%$, a specificity of $72.1 \%$, a PPV of $44.3 \%$, and an NPV of $88.6 \%$ in terms of predicting whether PBD was needed. The area under the ROC curve was 0.78 . Based on the current findings, preoperative TB of $218.75 \mu \mathrm{mol} / \mathrm{L}(12.4$ $\mathrm{mg} / \mathrm{dL}$ ) was an optimal reference point for deciding whether patients needed to undergo PBD.

\subsection{Comparison of charge}

The total charges and charges per day were shown in Table 3. The exchange rate was 6.6 RenMinBi (RMB, the official currency of the People's Republic of China) to 1 US dollar. The mean of total charges was higher in PBD group, but the mean total cost did not differ significantly between the two groups $(14,270 \pm 6,106$ US\$ vs. $12,793 \pm 8,621$ US $\$, p=0.588)$. Similarly, the cost per day did not differ significantly between the two groups $(622 \pm 231 \$ v s .719 \pm 316 \$, p=0.331)$.

\section{Discussion}

Currently, the clear consensusis that PBD is indicated for patients with cholangitis, hyperbilirubinemiainduced malnutrition, hepatic insufficiency, or renal insufficiency and patients undergoing preoperative anti-neoplastic therapy or PVE (11). The routine performance of $\mathrm{PBD}$ in other patients with $\mathrm{pCC}$ is still controversial. The current study focused on those patients. Surgical outcomes and hospital costs were compared for patients who underwent PBD and patients who did not, and ROC curve analysis was performed to find an optimal cut-off value for preoperative TB to indicate the need for PBD.

The current results indicated that the short-term surgical and postoperative outcomes of the group that directly underwent surgery were comparable to those of the group that underwent PBD. This finding is consistent with the results of previous studies and meta-analyses (12-14). Several studies have found that PBD increased the risk of postoperative complications and infections (15-17), but our data did not demonstrate this. In the current study, the disadvantages of PBD were a longer operating time and longer duration of hospitalization. However, the total cost of hospitalization for patients in PBD group did not increase. Although the current study noted no advantages of PBD in terms of shortterm surgical outcomes, attention must be paid to the potential advantages of PBD when treating patients with pCC. Nimura et al. noted that selective cholangiography through PTBD and/or ENBD allows precise preoperative staging of the disease (18).In addition, ERCP/ENBD can delineate the anatomy of the biliary system, determine the extent of bile duct involvement, and allow correct differentiation of malignant from benign lesions through cytology by brushings and biopsy, which are important in determining respectability and surgical management (19).These advantages cannot be ignored. Benign bile duct diseases are needed be excluded during the process of diagnosis, such as hepatolithiasis (20). Thus when the suspicion remains for $\mathrm{pCC}$, the PBD should be indicated due to the reasons mentioned above. Although the mortality rate was comparable between the two groups in the current study, no death was found in PBD group. Probably because of after performing PBD, some patients were found out that the tumor was unresectable and part of patients' liver function worsened before surgery was planned. Those patients were ultimately not eligible for surgery and did not undergo surgery. In addition, patients underwent PBD possess more time to evaluate the tumor, and a small part of tumors are progressing before surgery resulting in selecting other treatment. Regarding to vascular resection and reconstruction, a Belghiti's group showed that PBD increased the percentage of resectablitiy (21). The rate of vascular resection and reconstruction in PBD group was higher in their study, but in our study the rate was lower. In our center, as the value of resection and reconstruction of portal vein or hepatic artery (stage IV, AJCC $7^{\text {th }}$ staging system) is still controversial (22), so some of those patients at the current authors' Hospital wished to undergo chemotherapy rather than surgery for financial reasons. In addition, staging was more precise in the PBD group resulting in more patients with stage IV cancer were detected and ruled out in this study. This could explain the lower rate of vascular resection and reconstruction in the current study. 
Among patients with obstructive jaundice, PBD is the best way to reduce the bilirubin level to reverse cholestasis-associated liver dysfunction and impaired hepatic regeneration (23). However, there are no guidelines or indications regarding the level of bilirubin that warrants a patient undergoing PBD.A ROC curve was used in the current study to find the cut-off value for TB to indicate PBD. The classifier result should be a real value, so we excluded the patients underwent bad prognosis (death or heavy complications caused hospital stay over 30 days), and the remaining patients all had a good prognosis, and the cut-off value for TB in those patients can be regarded as a correct/real value. The cut-off value for preoperative TB was 218.75 $\mu \mathrm{mol} / \mathrm{L}(12.4 \mathrm{mg} / \mathrm{dL})$ based on the current findings. Su et al. reported that preoperative PTBD is advisable for pCC with severe jaundice $(>10 \mathrm{mg} / \mathrm{dL})(24)$. The cutoff value for TB in the current study was very close to the value reported by $\mathrm{Su}$ et al. A subgroup of patients with a TB level over $218.75 \mu \mathrm{mol} / \mathrm{L}$ was also analyzed in terms of those who underwent PBD and those that directly underwent surgery, but the outcomes for those patients were consistent with the outcomes for patients as a whole. Therefore, this cut off value of TB did not show any clinical benefit. We just emphasized that this point of value could be the best reference point to indicate the PBD based on our data. In the future, more clinical data need to be assembled and randomized controlled trials need to be conducted to resolve the controversy regarding PBD.

The current study had several limitations. First, incomplete data precluded the comparison of the two groups in terms of long-term outcomes and survival rates. Second, this study was retrospective, so selection bias potentially occurred and the study involved a small sample. Third, patient insurance differs widely among regions of China, so data on actual expenses could not be obtained. Only data on the total cost according to the hospital was available.

\section{Conclusion}

PBD has several advantages and disadvantages. In patients who did not undergo PVE and who did not have cholangitis, those that underwent PBD had comparable short-term outcomes to those that directly underwent surgery. When the diagnosis is still suspicious of pCC, PBD should be performed for the patients. The total hospital charges and charges per day were similar for the two groups. Based on our data, the optimal cut-off value for preoperative TB was $218.75 \mu \mathrm{mol} / \mathrm{L}(12.4$ $\mathrm{mg} / \mathrm{dL}$ ) to indicate the need for PBD in patients with pCC. These findings may offer some clues for further study. More RCTs and large-scale studies need to be conducted to resolve the controversy concerning PBD.

\section{References}

1. Klatskin G. Adenocarcinoma of the hepatic duct at its bifurcation within the porta hepatis: An unusual tumor with distinctive clinical and pathological features. Am J Med. 1965; 38:241-256.

2. Ito F, Agni R, Rettammel RJ, Been MJ, Cho CS, Mahvi DM, Rikkers LF, Weber SM. Resection of hilar cholangiocarcinoma: Concomitant liver resection decreases hepatic recurrence. Ann Surg. 2008; 248:273279.

3. Nagino M, Kamiya J, Uesaka K, Sano T, Yamamoto H, Hayakawa N, Kanai M, Nimura Y. Complications of hepatectomy for hilar cholangiocarcinoma. World J Surg. 2001; 25:1277-1283.

4. Kawasaki S, Imamura H, Kobayashi A, Noike T, Miwa S, Miyagawa S. Results of surgical resection for patients with hilar bile duct cancer: Application of extended hepatectomy after biliary drainage and hemihepatic portal vein embolization. Ann Surg. 2003; 238:84-92.

5. Cai Y, Cheng N, Ye H, Li F, Song P, Tang W. The current management of cholangiocarcinoma: A comparison of current guidelines. Biosci Trends. 2016; 10:92-102.

6. Iacono C, Ruzzenente A, Campagnaro T, Bortolasi L, Valdegamberi A, Guglielmi A. Role of preoperative biliary drainage in jaundiced patients who are candidates for pancreatoduodenectomy or hepatic resection: highlights and drawbacks. Ann Surg. 2013; 257:191-204.

7. Sewnath ME, Karsten TM, Prins MH, Rauws EJ, Obertop H, Gouma DJ. A meta-analysis on the efficacy of preoperative biliary drainage for tumors causing obstructive jaundice. Ann Surg. 2002; 236:17-27.

8. Bismuth H, Nakache R, Diamond T. Management strategies in resection for hilar cholangiocarcinoma. Ann Surg. 1992; 215:31-38.

9. Dindo D, Demartines N, Clavien PA. Classification of surgical complications: A new proposal with evaluation in a cohort of 6336 patients and results of a survey. Ann Surg. 2004; 240:205-213.

10. Japan LCSGo. General Rules for the Clinical and Pathological Study of Primary Liver Cancer. Tokyo: Kanehara, Tokyo, Japan, 2000.

11. Mansour JC, Aloia TA, Crane CH, Heimbach JK, Nagino M, Vauthey JN. Hilar cholangiocarcinoma: Expert consensus statement. HPB (Oxford). 2015; 17:691-699.

12. Farges O, Regimbeau JM, Fuks D, Le Treut YP, Cherqui D, Bachellier P, Mabrut JY, Adham M, Pruvot FR, Gigot JF. Multicentre European study of preoperative biliary drainage for hilar cholangiocarcinoma. Br J Surg. 2013; 100:274-283.

13. Liu F, Li Y, Wei Y, Li B. Preoperative biliary drainage before resection for hilar cholangiocarcinoma: Whether or not? A systematic review. Dig Dis Sci. 2011; 56:663672.

14. Ribero D, Zimmitti G, Aloia TA, Shindoh J, Forchino F, Amisano M, Passot G, Ferrero A, Vauthey JN. Preoperative cholangitis and future liver remnant volume determine the risk of liver failure in patients undergoing resection for hilar cholangiocarcinoma. J Am Coll Surg. 2016; 223:87-97.

15. Ercolani G, Zanello M, Grazi GL, Cescon M, Ravaioli M, Del Gaudio M, Vetrone G, Cucchetti A, Brandi G, Ramacciato G, Pinna AD. Changes in the surgical approach to hilar cholangiocarcinoma during an 18year period in a Western single center. J Hepatobiliary 
Pancreat Sci. 2010; 17:329-337.

16. Ferrero A, Lo Tesoriere R, Vigano L, Caggiano L, Sgotto E, Capussotti L. Preoperative biliary drainage increases infectious complications after hepatectomy for proximal bile duct tumor obstruction. World J Surg. 2009; 33:318325 .

17. Hochwald SN, Burke EC, Jarnagin WR, Fong Y, Blumgart LH. Association of preoperative biliary stenting with increased postoperative infectious complications in proximal cholangiocarcinoma. Arch Surg. 1999; 134:261-266.

18. Nimura Y. Preoperative biliary drainage before resection for cholangiocarcinoma (Pro). HPB (Oxford). 2008; 10:130-133.

19. Alvaro D, Cannizzaro R, Labianca R, Valvo F, Farinati F, Italian Society of G, Italian Association of Hospital G, Italian Association of Medical O, Italian Association of Oncological R. Cholangiocarcinoma: A position paper by the Italian Society of Gastroenterology (SIGE), the Italian Association of Hospital Gastroenterology (AIGO), the Italian Association of Medical Oncology (AIOM) and the Italian Association of Oncological Radiotherapy (AIRO). Dig Liver Dis. 2010; 42:831-838.
20. Feng X, Zheng S, Xia F, Ma K, Wang S, Bie P, Dong J. Classification and management of hepatolithiasis: A high-volume, single-center's experience. Intractable Rare Dis Res. 2012; 1:151-156.

21. Belghiti J, Ogata S. Preoperative optimization of the liver for resection in patients with hilar cholangiocarcinoma. HPB (Oxford). 2005; 7:252-253.

22. Miyazaki M, Yoshitomi H, Miyakawa S, et al. Clinical practice guidelines for the management of biliary tract cancers 2015: 2nd English edition. J Hepatobiliary Pancreat Sci. 2015; 22:249-273.

23. van der Gaag NA, Kloek JJ, de Castro SM, Busch OR, van Gulik TM, Gouma DJ. Preoperative biliary drainage in patients with obstructive jaundice: History and current status. J Gastrointest Surg. 2009; 13:814-820.

24. Su CH, Tsay SH, Wu CC, Shyr YM, King KL, Lee $\mathrm{CH}$, Lui WY, Liu TJ, P'Eng F K. Factors influencing postoperative morbidity, mortality, and survival after resection for hilar cholangiocarcinoma. Ann Surg. 1996; 223:384-394.

(Received January 23, 2017; Revised May 5, 2017; Accepted May 15, 2017) 